\title{
Listado actualizado de la herpetofauna de la Reserva de la Biosfera Barranca de Metztitlán, Hidalgo, México
}

\author{
Sharon Yedid Valdez-Rentería ${ }^{1}$, Leonardo Fernández-Badillo ${ }^{2,3^{*}}$, \\ Cristian Raúl Olvera-Olvera ${ }^{4}$, Guillermo Sánchez-Martínez ${ }^{3,5,6}$ e Irene Goyenechea 7
}

\section{Resumen}

La Reserva de la Biósfera Barranca de Metztitlán (RBBM), es el área natural protegida (ANP) más grande del estado de Hidalgo. Aunque cuenta con estudios herpetofaunísticos previos, éstos no incluyen el total de especies que en ella habitan. Por lo anterior, el objetivo de este estudio fue actualizar el inventario de este grupo de vertebrados donde se compiló la información de siete años de trabajo de campo realizado en siete de los ocho municipios que abarcan la reserva, así como la consulta de bases de datos y literatura. El listado que se presenta incluye sesenta y nueve especies, de las cuales nueve representan nuevos registros para esta ANP. La información anterior permite considerar a la RBBM como el ANP de Hidalgo de mayor importancia en riqueza y conservación de este grupo de vertebrados.

Palabras clave. RBBM, inventario, herpetofaunístico, ANP, conservación.

\footnotetext{
${ }^{1}$ Domicilio conocido, Tezontepec de Aldama, Hidalgo, México.

${ }^{2}$ Centro de Investigaciones Biológicas, Universidad Autónoma del Estado de Hidalgo. Ciudad del Conocimiento, Carretera Pachuca-Tulancingo Km 4.5, 42184 Pachuca, Hidalgo, México.

${ }^{3}$ Predio intensivo de manejo de vida silvestre X-plora Reptilia, Km 65 Carretera México-Tampico s/n, Pilas y granadas, 43350, Metztitlán, Hidalgo, México.

${ }^{4}$ Laboratorio de Morfología Animal, Centro de Investigaciones Biológicas, Universidad Autónoma de Estado de Hidalgo, Ciudad del conocimiento, Carretera Pachuca-Tulancingo Km 4.5, 42184 Pachuca, Hidalgo, México.

${ }^{5}$ Domicilio conocido, Acalome, 43359, Metztitlán, Hidalgo, México.

${ }^{6}$ UMA Santana, km 65 Carretera México-Tampico s/n, Pilas y Granadas, 43350, Metztitlán, Hidalgo, México.

${ }^{7}$ Laboratorio de Sistemática Molecular, Centro de Investigaciones Biológicas, Universidad Autónoma de Estado de Hidalgo, Ciudad del conocimiento, Carretera Pachuca-Tulancingo Km 4.5, 42184 Pachuca, Hidalgo, México.

Correspondencia: fernandezbadillo80@gmail.com*
} 


\section{Abstract}

Barranca de Metztitlán Biosphere Reserve (RBBM, for its acronym in Spanish) is the largest protected natural area (PNA) in the State of Hidalgo. Although previous herpetofaunistic studies have been conducted in this area, they do not include the total number of species that inhabit the reserve. Therefore, with the purpose of updating the inventory on this vertebrate group, we compiled data from seven years of field work performed in seven of the eight municipalities that comprise this PNA, as well as databases and literature query. The updated list includes sixty nine species, of which nine represent new records. The above information allows us to consider RBBM as the most important PNA of Hidalgo in terms of species richness and conservation priority for this group of vertebrates.

Key words. RBMM, inventory, herpetofaunistic PNA, conservation

\section{Introducción}

Las Áreas Naturales Protegidas (ANP), son la mejor herramienta con la que cuenta México para conservar la biodiversidad y los servicios ambientales que esta proporciona a la sociedad (Bezaury-Creel et al., 2009). Para el estado de Hidalgo, existen cinco ANP de carácter federal, el Área de Protección de Recursos Naturales Cuenca Hidrológica del Río Necaxa (APRNCHRN), el Parque Nacional el Chico (PNCH), el Parque Nacional los Mármoles (PNLM), el Parque Nacional Tula (PNT) y la Reserva de la Biosfera Barranca de Metztitlán (RBBM) (Durán y Larios, 2001; Cruz-Elizalde et al., 2015).

Específicamente, la RBBM, es el ANP más grande del estado, con una extensión de 960.43 km², y debido a su ubicación geográfica y a las características geológicas de la región, en ella existe una gran diversidad tanto de tipos de vegetación (Vite-Silva et al., 2010), como de vertebrados. Por ejemplo, para el caso de las aves, se han registrado 271 especies (Ortiz-Pulido et al., 2010) y 75 de mamíferos (Rojas-Martínez et al., 2017). En cuanto a la herpetofauna, existen diversos trabajos que difieren en el número de especies presentes dentro de la reserva. Por ejemplo, el plan de manejo de la RBBM (CONANP, 2003) enlista 33 especies, mientras que Vite-Silva et al. (2010) registran 38 
especies (26 de ellas registradas en campo y 12 en fuentes bibliográficas).

Por otro lado, recientemente Cruz-Elizalde et al. (2015), evaluaron la efectividad de tres ANP de Hidalgo (PNCH, PNLM y RBBM) en la conservación de la herpetofauna, a partir únicamente de datos de campo, sin incluir registros bibliográficos, y enlistan para la RBBM un total de 28 especies, que corresponden a cinco anfibios y 23 reptiles (miembros no voladores de la clase Sauropsida).

Aunado a lo anterior, existen varios registros bibliográficos (Mendoza-Quijano y Smith, 1993; Hernández-Pérez, 1997; Altamirano-Álvarez et al., 1999; Gelover-Alfaro et al., 2000; CamposRodríguez y López-Vidal, 2004; Goyenechea et al., 2010; Vite-Silva, 2010; Ramírez-Bautista et al., 2014; Fernández-Badillo y Flores-Vargas, 2015), que no fueron incluidos en los listados presentados por Vite-Silva et al. (2010) y Cruz-Elizalde et al. (2015), además, se han publicado últimamente nuevos registros para la reserva (Morales-Capellán et al., 2016; Fernández-Badillo et al., 2016a, b, d; Fernández-Badillo et al., 2017 a, b, c; Olvera-Olvera y Iturbe-Morgado, 2017) y se cuenta asimismo con datos de distribución incluidos en trabajos recientes (Lemos-Espinal y Dixon, 2016; Fernández-Badillo et al., 2017c) que incrementan el listado herpetofaunístico de esta área. Sin embargo, hasta el momento dicha información se encuentra dispersa y no se cuenta con un inventario actualizado y completo.

Lo anterior es relevante, ya que tal como lo mencionan Ortiz-Pulido et al. (2010), el buen funcionamiento de una área natural protegida depende en gran medida de los conocimientos que se tengan sobre las especies animales y vegetales presentes en ésta. De este modo, es importante contar con inventarios lo más completos posible para conocer la riqueza total del área y desarrollar así estrategias de conservación que incluyan a la mayoría de las especies (Dirzo y Raven, 1994).

Por lo anterior el objetivo principal del presente trabajo fue elaborar una lista actualizada de la herpetofauna que habita en la RBBM, a partir de la compilación de registros obtenidos mediante trabajo de campo, revisión bibliográfica y bases de datos.

\section{Materiales y métodos}

\section{Área de estudio}

La RBBM, se ubica en la parte centro de Hidalgo, se encuentra dentro de los límites de ocho municipios (Metztitlán, San Agustín Metzquititlán, Zacualtipán de Ángeles, Huasca de Ocampo, 
Atotonilco el Grande, Metepec, Eloxochitlán y Acatlán). En ella convergen tres provincias biogeográficas: la Sierra Madre Oriental, la Faja Volcánica Transmexicana y el Altiplano Mexicano, lo que permite la presencia de seis tipos de vegetación: selva baja caducifolia, matorral xerófilo, matorral submontano, bosque de Juniperus, bosque de encino y bosque de pino-encino (CONANP, 2003). El intervalo altitudinal de la reserva abarca desde los 900 hasta los 2,500 m y se presentan dos tipos de clima. En la porción norte y norte-centro predomina el clima seco semicálido con lluvias en verano, en tanto que en la parte centro-sur y sur, predomina el clima semiseco templado con lluvias en verano (CONANP, 2003).

\section{Trabajo de campo}

Se realizaron salidas esporádicas en los años 2010, 2011, 2016 y 2017, así como otras llevadas a cabo mediante un muestreo sistemático de marzo de 2014 a julio de 2015, en 22 localidades pertenecientes a siete municipios (Acatlán, Atotonilco el Grande, Eloxochitlán, Huasca de Ocampo, Metztitlán, San Agustín Metzquititlán y Zacualtipán; Fig. 1). En dicho periodo se realizaron 18 salidas al campo con cinco personas, con una duración de tres días y un esfuerzo de muestreo de ocho horas diarias, lo que representa un esfuerzo de muestreo total de (2,160 horas-persona); el cual se analizó mediante una curva de acumulación de especies con el estimador no paramétrico Bootstrap (Moreno, 2001), en el programa EstimateS 9. Este estimador se considera adecuado para la herpetofauna, ya que no se ve sesgado por las especies raras, lo cual es común en las comunidades herpetofaunísticas (Carvajal-Cogollo y Urbina-Cardona, 2008).

El muestreo se realizó mediante la técnica de búsqueda directa no restringida (Manzanilla y Pefaúr, 2010) con recorridos al azar diurnos y nocturnos, en los cuales se revisaron todos los microhábitats posibles, para localizar a los organismos. A partir de las recomendaciones de otros autores (Muñoz-Alonso, 1998; Barragán-Vázquez, 2006; Fernández-Badillo y Goyenechea, 2010; Medina-Aguilar et al., 2011) se recolectaron únicamente los ejemplares que no pudieron ser identificados en campo y fueron trasladados al laboratorio de Colecciones Húmedas del Centro de Investigaciones Biológicas de la Universidad Autónoma de Hidalgo (CH-CIB) para su posterior determinación taxonómica, bajo la extensión de permiso de colecta FAUT-0052 a nombre de Irene Goyenechea. Los organismos se determinaron tanto en campo como en laboratorio con el apoyo de claves taxonómicas y guías de campo (Smith y Taylor, 1966; Lynch, 1970; Flores-Villela 
et al., 1995; Rossman, 1996; Duellman, 2001; Canseco-Márquez y Gutiérrez-Mayén, 2010; Dixon y Lemos-Espinal, 2010).

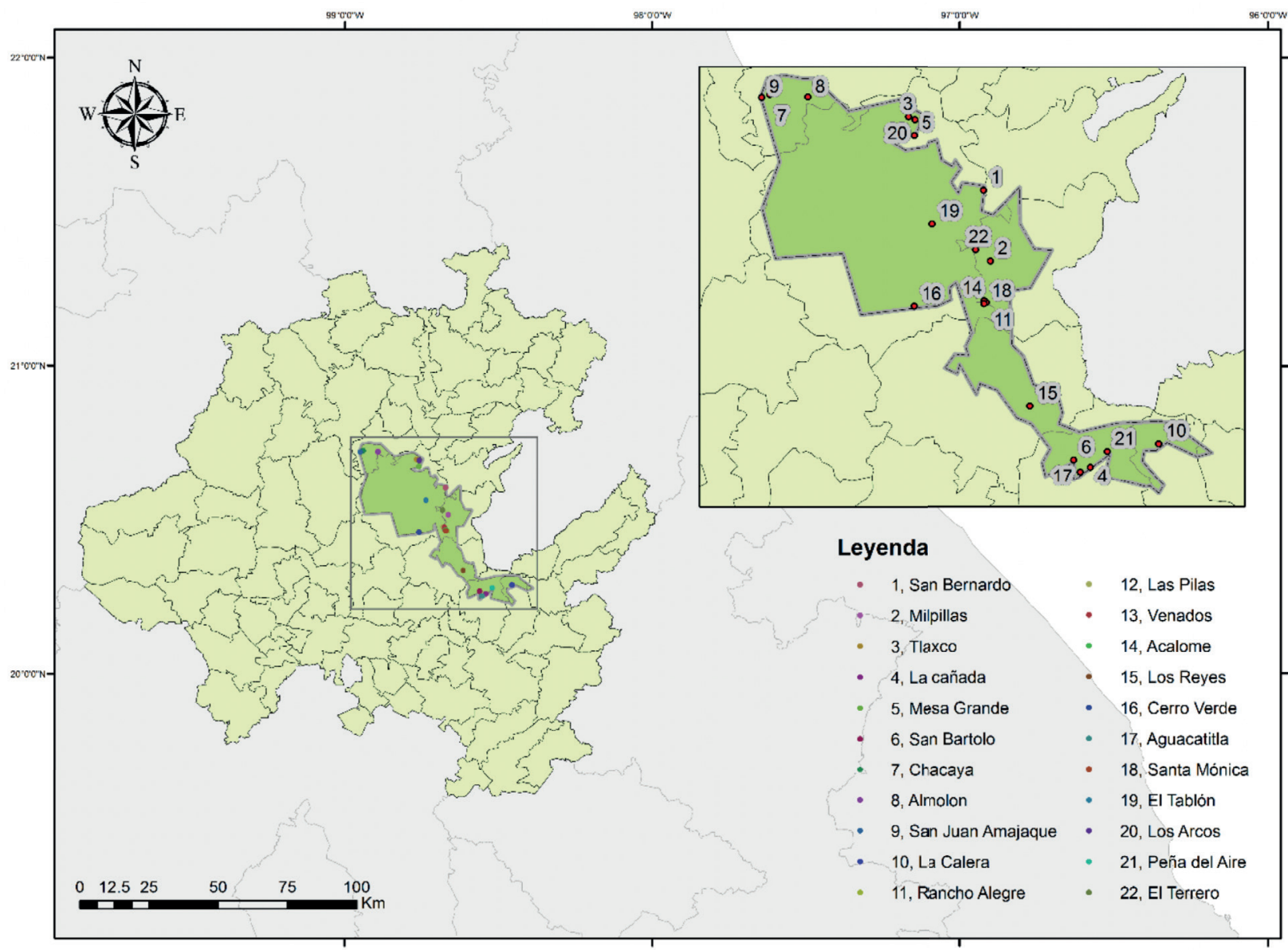

Figura 1. Mapa en el que se muestra el polígono de la RBBM así como las localidades que fueron muestreadas durante el muestreo sistemático.

\section{Revisión bibliográfica y de bases de datos}

Se revisaron distintos trabajos bibliográficos (Mendoza-Quijano, 1990; Mendoza-Quijano y Smith, 1993; Hernández-Pérez, 1997; Altamirano-Álvarez et al., 1999; Gelover-Alfaro et al., 2000; CONANP, 2003; Campos-Rodríguez y López-Vidal, 2004; Vite-Silva, 2008; Goyenechea et al., 2010; RamírezBautista et al., 2010;2014; Vite-Silva et al., 2010; Fernández-Badillo et al., 2011; Cruz-Elizalde et al., 2015; Fernández-Badillo y Flores-Vargas, 2015; Fernández-Badillo et al., 2016a, b, c, d; 2017a; b; Lemos-Espinal y Dixon, 2016; Morales-Capellán et al., 2016; Fernández-Badillo et al., 2017a,b; Olvera-Olvera e Iturbe-Morgado, 2017), así como colecciones científicas (CH-CIB-UAEH, MZFCUNAM, CNAR-UNAM) y bases de datos en línea (HERPNET, REMIB-CONABIO). A partir de toda la información anterior, se eliminaron los registros de colecciones científicas y bases de datos 
en línea que no pudieron ser corroborados en campo o en fuentes bibliográficas, y cuya presencia para la reserva resultaba dudosa debido a que no coincidía con la distribución conocida de la especie ni con su tipo de hábitat. Asimismo, algunos registros bibliográficos dudosos se eliminaron y se verificó la identidad taxonómica de los especímenes recolectados dentro de la RBBM (Vite-Silva et al., 2010) y depositados en la Colección Herpetológica del Centro de Investigaciones Biológicas (CH-CIB) de la Universidad Autónoma del Estado de Hidalgo.

\section{Compilación del listado de especies}

Se elaboró un listado herpetofaunístico para la Reserva de la Biosfera Barranca de Metztitlán en el que se eliminaron sinonimias y se actualizó la taxonomía con base en los trabajos de Mulcahy y Mendelson (2000); Wilson y Johnson et al. (2010); Rovito et al. (2015); Duellman et al. (2016); Heimes, (2016). En el listado se indica, el estado de conservación de las especies referido en la NOM-SEMARNAT-059-2010 (SEMARNAT, 2010), IUCN, así como su distribución (endémicas o no a México), lo cual se consultó a partir del uso de la aplicación "validación de especies" del portal http:/ / www.enciclovida.com/validaciones (acceso 30 enero 2018).

\section{Resultados y discusión}

Se obtuvo una listado herpetofaunístico de 69 especies para la RBBM, agrupadas en 22 familias y 44 géneros. Nueve especies (cuatro anfibios y cinco reptiles) corresponden a nuevos registros para la zona, las cuales no habían sido encontradas en estudios previos (Vite-Silva et al., 2010; RamírezBautista et al., 2014; Cruz-Elizalde et al., 2015; Lemos-Espinal y Dixon, 2016; Fig. 2; Anexo 1, 2 a, b). De las 69 especies registradas, 61 (13 anfibios y 48 reptiles) se registraron durante el trabajo de campo. Para el caso de los anfibios (Clase Amphibia), se registraron 14 especies agrupadas en dos órdenes (una de Caudata y 13 de Anura), siete familias y nueve géneros. La mayor riqueza se presentó en la familia Hylidae con cuatro especies; los géneros más ricos de anfibios fueron Dryophytes y Rana con tres especies cada uno (Anexo 1). Para los reptiles, se registraron 55 especies agrupadas en dos órdenes (una de Testudines y 54 de Squamata), 15 familias y 35 géneros. La familia con mayor número de especies fue Colubridae con 14, en tanto el género más rico fue Sceloporus con siete especies (Anexo $2 \mathrm{a}, \mathrm{b}$ ).

Con base en la evaluación del esfuerzo de muestreo de las salidas sistemáticas realizadas de 
marzo de 2012 a julio de 2015, se obtuvo una completitud del inventario del 92.7\% para anfibios y del $93.9 \%$ para reptiles (Fig. 3a, b). En este sentido, de acuerdo con Pineda-López y VerdúFranco (2013), cuando el porcentaje de las especies observadas es mayor al 80\% de la riqueza total estimada, se considera que un inventario está razonablemente completo.

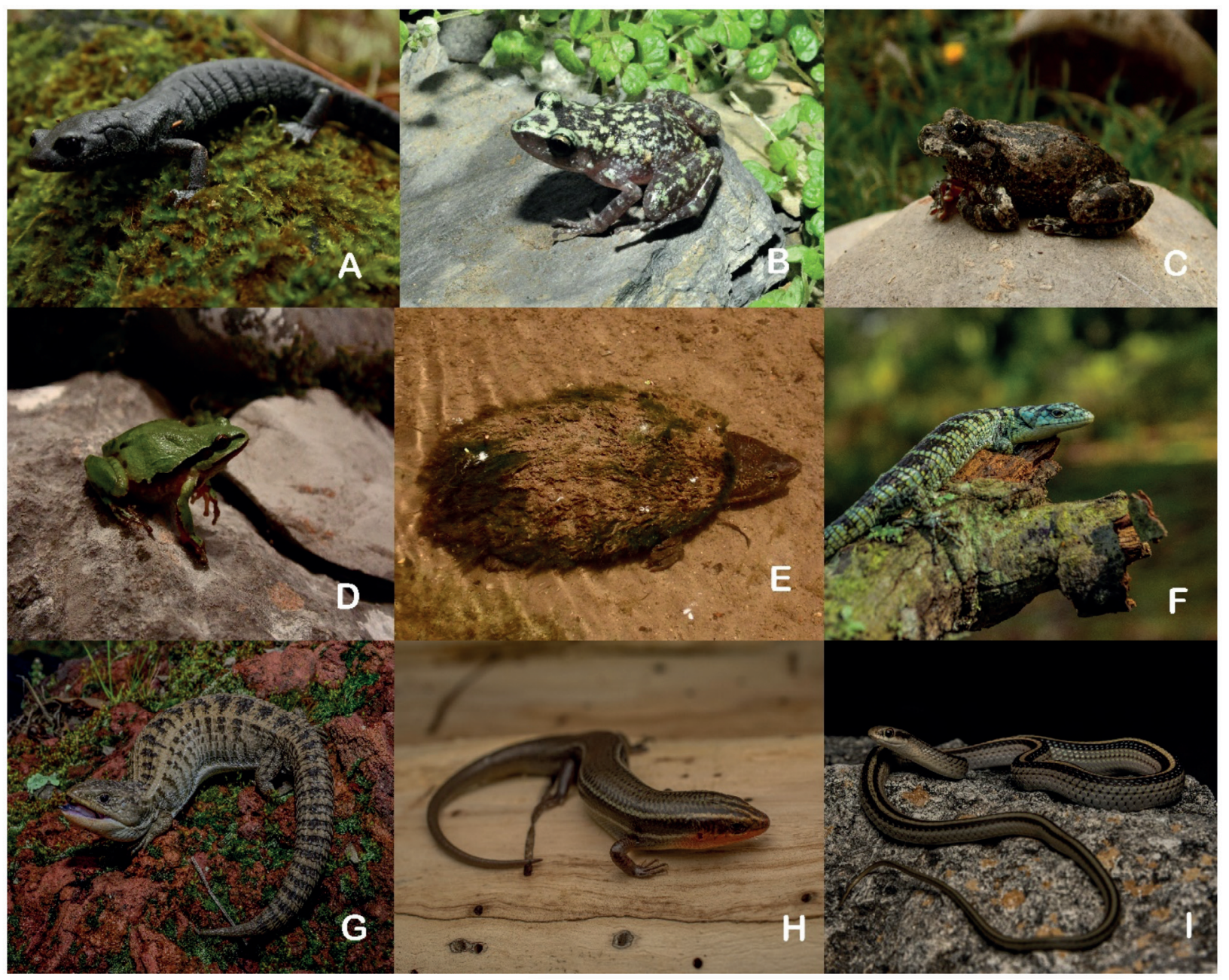

Figura 2. Fotografías de las especies que representan nuevos registros en la RBBM. A) Aquiloeurycea cephalica, B) Eleutherodactylus verrucipes, C) Dryophytes arenicolor, D) D. plicatus, E) Kinosternon hirtipes, F) Abronia taeniata, G) Barisia imbricata, H) Plestiodon tetragrammus, I) Salvadora bairdi. Fotografías: Sharon Yedid Valdez-Rentería, Cristian R. Olvera-Olvera y Leonardo Fernández-Badillo.

A partir de la revisión de los ejemplares depositados en la $\mathrm{CH}-\mathrm{CIB}$, se corrigió la ubicación taxonómica de dos ejemplares mencionados por Vite-Silva et al. (2010; CH-CIB 1850, 1934) como Crotalus triseriatus (Wagler, 1830) y Geophis semidoliatus (Duméril, Bibron y Duméril, 1854), los cuales de acuerdo con los trabajos de Campbell y Lamar (2004) y Heimes (2016), corresponden a las especies Crotalus aquilus (Klauber, 1952) y Geophis latifrontalis (Garman, 1883) respectivamente.

Por otro lado, se consideró que los registros previos de Incilius valliceps (Wiegmann, 1833) para 
la RBBM (CONANP, 2003; Vite-Silva et al., 2010, Ramírez-Bautista et al., 2014; Cruz-Elizalde et al., 2015; Lemos-Espinal y Dixon, 2016; Valdez-Rentería, 2017), corresponden a la especie I. nebulifer (Girard, 1854) con base en el trabajo de Mulcahy y Mendelson (2000).

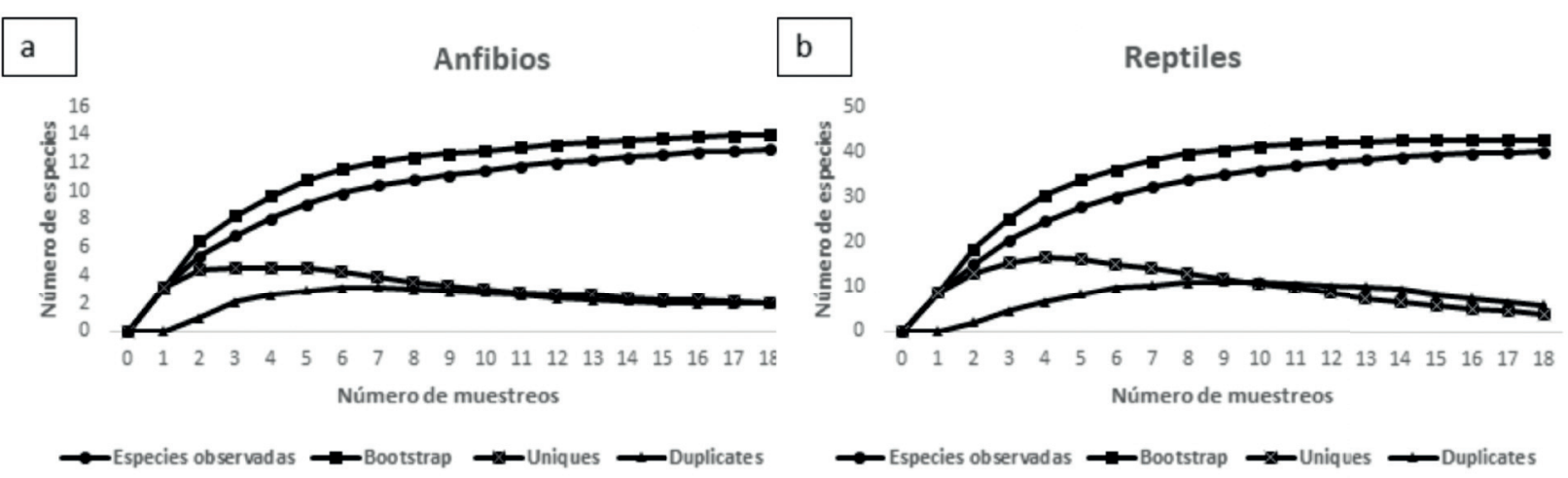

Figura 3. Curvas de acumulación; a) anfibios, b) reptiles.

Se descartó la presencia de Epictia goudotti referida por Ramírez-Bautista et al. (2014) para el municipio de Metztitlán, ya que los autores citan como fuente los trabajos de Ramírez-Bautista et al. (2010) y Pampa-Ramírez (2010). Sin embargo, en dichos trabajos no se menciona ningún registro de esa especie en Metztitlán. Además, tampoco se encuentra considerada en trabajos posteriores (Vite-Silva et al., 2010; Cruz-Elizalde et al., 2015). También se descartó la presencia de Plectrohyla arborescadens (Taylor, 1839) registrada por Lemos-Espinal y Dixon (2016) en el municipio de Eloxochitlán, ya que el sitio de la coordenada referida, no concuerda con las características del hábitat mencionado por estos autores, quienes indican que esta especie habita bosques nublados o bosque fríos de pino-encino, mientras que la coordenada referida se encuentra en un área de matorral xerófilo, por lo que es probable que exista un error en la georeferencia del lugar de recolecta de esta especie.

Es probable que aún puedan registrarse otras especies dentro de la RBBM, debido a que no se han explorado algunas barrancas de difícil acceso. Por la cercanía con los bosques de la Sierra Madre Oriental, es probable la presencia de Chiropterotriton chondrostega (Taylor, 1941), Isthmura bellii (Gray, 1850) y Thamnophis sumichrasti (Cope, 1866) (Mendoza-Quijano, 1990; Lemos-Espinal y Dixon, 2016). Cabe mencionar que Ambystoma velasci (Dugès, 1888) fue registrada por LemosEspinal y Dixon (2016), en la localidad de Santa Mónica, sin embargo, los pobladores de zonas cercanas a la localidad de registro no reconocen haber visto ningún animal similar a esta especie. 
La riqueza encontrada en el presente estudio (69 especies), incrementó las especies registradas por Vite-Silva et al. (2010) y Cruz-Elizalde et al. (2015) en un $44.9 \%$ y en un 59.5\% respectivamente para la RBBM. De acuerdo con Ramírez-Bautista et al. (2014), la herpetofauna de Hidalgo está compuesta por 183 especies (53 anfibios y 130 reptiles); con base en estos datos, en la RBBM se encuentran el $26.4 \%$ de los anfibios y al $42.3 \%$ de los reptiles del estado. Recientemente, CruzElizalde et al. (2015) analizaron la efectividad de tres ANP de Hidalgo (PNCH, PNLM y RBBM) para la conservación de la herpetofauna, con base en listados obtenidos a partir de muestreos en campo, sin incluir registros bibliográficos ni toda la información existente, y concluyeron que la RBBM es la tercera en importancia para la conservación de anfibios con cinco especies y la segunda en conservación de reptiles con 23 especies, y clasificaron al PNLM como el más importante para ambos grupos, con un total de 36 especies (nueve anfibios y 27 reptiles). Sin embargo, los resultados obtenidos en el presente trabajo demuestran que la RBBM, en comparación con el PNCH y PNLM, alberga una mayor riqueza de especies y debe considerarse como el área natural protegida con mayor importancia para la conservación de la herpetofauna en Hidalgo. Lo anterior hace relevante la importancia de contar con inventarios lo más completos posibles, así como un sistema de monitoreo constante de las poblaciones de anfibios y reptiles que habitan la reserva, antes de sugerir o tomar decisiones en términos de priorización de áreas. Inclusive, aún considerando datos recientes y actualizados de la herpetofauna del PNLM (61 especies; Flores-Hernández y Fernández-Badillo, en preparación), este es superado en número de especies por la RBBM. Por otro lado, es importante resaltar que el esfuerzo de muestreo realizado en las salidas sistemáticas del presente estudio (2,160 horas-persona) fue considerablemente mayor al empleado en trabajos previos (Vite-Silva et al., 2010 con 528 horas-persona y Cruz-Elizalde et al., 2015 con 960 horaspersona), lo que sin duda permitió el registro de un mayor número de especies, además de que los valores de completitud del inventario fueron superiores al 90\%. Lo anterior concuerda con lo que habían sugerido Vite-Silva et al. (2010), quienes consideraron que un esfuerzo de muestreo mayor en ciertas áreas de la reserva, podría contribuir al registro de una mayor riqueza de especies.

La riqueza herpetofaunística encontrada en la RBBM, puede explicarse por varios factores. Primero, esta reserva es un área geográfica con una importante heterogeneidad en tipos de vegetación e intervalos altitudinales variables, que propician la presencia de una alta diversidad de flora y fauna (Rojas-Martínez et al., 2017), lo cual se debe en parte a su ubicación geográfica 
entre distintas regiones y provincias biogeográficas (CONANP, 2003; Vite-Silva et al., 2010). Por otro lado, la RBBM se encuentra dentro de la porción hidalguense de la ecorregión del Matorral de la Meseta Central (Olson et al., 2001), y de acuerdo con Fernández-Badillo et al. (2016 c), la riqueza de esta zona árida de Hidalgo, se debe a que en ella convergen especies de afinidad tropical como Drymarchon melanurus (Duméril, Bibron y Duméril, 1854), Hemidactylus frenatus (Duméril y Bibron, 1836), Nerodia rhombifer (Hallowell, 1852), Oxybelis aeneus (Wagler, 1824), Rhinella horribilis (Linnaeus, 1758), Sceloporus variabilis (Wiegmann, 1834), T. proximus (Say, 1823) y Tropidodipsas sartorii (Cope, 1863), de afinidad templada como Eleutherodactylus verrucipes (Cope, 1885), Ficimia hardyi (Mendoza-Quijano y Smith, 1993), Geophis latifrontalis, Lepidophyma occulor (Smith, 1942), Rhadinaea gaigeae (Bailey, 1937) y Storeria hidalgoensis (Taylor, 1942) y especies de amplia distribución en el desierto Chihuahuense como Crotalus atrox (Baird y Girard, 1853).

Del total de especies registradas (69), únicamente 28 se encuentran bajo alguna categoría de riesgo de acuerdo con la NOM-SEMARNAT-059-2010 (SEMARNAT, 2010; ver Anexo 1, 2a y b); de estas, nueve están amenazadas (13.04\%) y 21 bajo protección especial (27.5\%). De acuerdo con la IUCN, 61 especies se encuentran bajo alguna categoría (ver Anexo 1), dos casi amenazadas $(2.9 \%)$, tres con datos insuficientes $(4.3 \%)$, una especie en peligro de extinción (1.4\%), tres especies vulnerables (4.3\%) y 52 bajo preocupación menor (75.3\%). Aunque no se registró ninguna especie endémica para la RBBM, 38 (55.1\%) de las 69 especies presentes en la reserva, son endémicas a México.

Los resultados aquí presentados, en comparación con lo registrado en trabajos previos (CONANP 2003, Vite-Silva et al., 2010; Cruz-Elizalde et al., 2015), brindan una mayor información sobre la riqueza herpetofaunística que habita dentro de la RBBM, la cual debe ser incluida en su totalidad dentro del plan de manejo de la reserva y con ello favorecer el planteamiento y desarrollo de mejores estrategias de uso, manejo y conservación, puesto que actualmente sólo 29 especies están incluidas en dicho plan (CONANP, 2003), lo que equivale al 39.7\% de la herpetofauna total presente en la RBBM.

\section{Conclusiones}

La alta riqueza herpetofaunística de la RBBM (69 especies), le confiere a esta ANP relevancia en 
términos de conservación para este grupo de vertebrados en el estado de Hidalgo, por lo que es indispensable actualizar el plan de manejo de la reserva, para incluir a todas las especies y realizar estudios para conocer el estado de las poblaciones, sobre todo de las especies amenazadas y/o en peligro, y desarrollar proyectos para su protección y conservación. Así mismo, es necesario continuar con las investigaciones en esta ANP, ya que debido a sus características y su extensión territorial, es posible que aún haya especies por encontrar.

\section{Agradecimientos}

Los autores agradecen a las autoridades de la CONANP a cargo de la Reserva de la Biosfera Barranca de Metztitlán y a la brigada comunitaria de la RBBM por permitirnos trabajar dentro de la Reserva y el apoyo en el trabajo de campo. Merecen un agradecimiento especial las personas y los delegados de las comunidades de la Mesa Grande, Aguacatitla, Chacaya, el Tablón, Peña del aire, el Terrero, San Bernardo, la Calera, los Reyes, San Juan Amajaque, Acalome, la Cañada, Rancho Alegre y Cerro Verde, por permitirnos muestrear y por su hospitalidad. A José Carlos Iturbe Morgado, Francisco Javier Callejas Jiménez, Nallely Morales Capellán, Martha Beatriz Ramírez Cruz, Ricardo López Mata, Mariana Edith Hernández Ortega, Ferdinand Torres Ángeles, Rosa Elena Torres Estrada, Juan Alfonso Hernández Melo y Eduardo Asturiano, por el apoyo en el trabajo de campo. A Luis Canseco Márquez por el apoyo en la determinación taxonómica de algunas especies. A Hublester Domínguez por apoyarnos en la realización del mapa utilizado en este trabajo. A Aurelio Ramírez-Bautista y Raciel Cruz-Elizalde por la información proporcionada al respecto de ejemplares recolectados en la RBBM. A los encargados de las colecciones de la CNAR-UNAM, CH-CIB-UAEH y al MZFC-UNAM (Víctor Hugo Reynoso, Adrián Nieto Montes de Oca, respectivamente) por permitirnos la revisión de las bases de datos, a OLMEC por el equipo herpetológico proporcionado y al proyecto “Diversidad Biológica del Estado de Hidalgo (tercera etapa) FOMIX-CONACyT-HGO-2012-191908." 


\section{Literatura citada}

Altamirano-Álvarez T., F. Mendoza-Quijano, M. Soriano-Sarabia, A. Gelover-Alfaro, E. Hernández-

Pérez. 1999. Anfibios y reptiles de la Barranca de Metztitlán. Revista de Zoología 10: 1-5.

Barragán-Vázquez, M.R., 2006. Anfibios y reptiles de dos ambientes en la sierra de Boca del Cerro, Tenosique, Tabasco, México. Kuxulkab’ 7 (14): 65-74.

Bezaury-Creel, J., D. Gutiérrez-Carbonel, J. F. Remolina, J. J. Pérez, J. González-Cano, N. Betancourt, M. Trigo, J. Antele, R. Frías, J. de la Maza, V. Sánchez-Cordero, F. Figueroa, P. Illodi, M. Linaje, C. A. Sifuentes, R. González-Montagut, H. A. López-López, A. Durán-Fuentes, R. G. de la Maza, S. Anta-Fonsecca y G. Sánchez. 2009. Áreas naturales protegidas y desarrollo social en México. En: Capital natural de México, Vol. II. pp. 385-431. En: Estado de conservación y tendencias de cambio. Comisión Nacional para el Conocimiento y Uso de la Biodiversidad (CONABIO). México. 821 pp.

Campos-Rodríguez, J. L., y J. C. López-Vidal. 2004. Gerrhonotus liocephalus (Texas Alligator Lizard). Geographic Distribution. Herpetological Review 35 (2): 187.

Canseco-Márquez, L. y M. G. Gutiérrez-Mayén. 2010. Anfibios y reptiles del valle de TehuacánCuicatlán. Comisión Nacional para el Conocimiento y Uso de la Biodiversidad (CONABIO). México. 292 pp.

Carvajal-Cogollo, J. E., y J. N. Urbina-Cardona. 2008. Patrones de diversidad y composición de reptiles en fragmentos de bosque seco tropical en Córdoba, Colombia. Tropical Conservation Science 1(4): 397-416.

Comisión Nacional de Áreas Protegidas (CONANP). 2003. Programa de Manejo de la Reserva de la Biosfera Barranca de Metztitlán, México. Comisión Nacional de Áreas Protegidas (CONANP). México. 209 pp.

Cruz-Elizalde, R, A. Ramírez-Bautista, L.D. Wilson y U. Hernández-Salinas. 2015. Effectiveness of protected areas in herpetofaunal conservation in Hidalgo, Mexico. Herpetological journal 25: 4148.

Dirzo, R. y P.H. Raven. 1994. Un inventario biológico para México. Botánica 55: 29-34.

Duellman, W. 2001. The Hylid frogs of Middle America. Society for the Study of Amphibians and 
Reptiles. Ithaca, Nueva York, EUA. 1158 pp.

Durán, A. y J. A. Larios. 2001. Ordenamiento ecológico territorial del estado de Hidalgo. Instituto Nacional de Estadística y Geografía (INEGI). México. 85 pp.

Fernández-Badillo, L. e I. Goyenechea. 2010. Anfibios y reptiles del Valle del Mezquital, Hidalgo, México. Revista Mexicana de Biodiversidad 81 (3): 705-712.

Fernández-Badillo, L., N. Morales-Capellán e I. Goyenechea-Mayer. 2011. Serpientes venenosas del Estado de Hidalgo. Universidad Autónoma de Estado de Hidalgo. Pachuca, Hidalgo. 90 pp.

Fernández-Badillo, L., D. R. Aguillón-Gutiérrez, S. Y. Valdez-Rentería, J. A. Hernández-Melo, C.R. Olvera-Olvera, F.J. Callejas-Jiménez, M. Hernández-Ramos, J.C. Iturbe-Morgado, F. Torres-Angeles e I. Reaño-Hernández. 2016a. First Records for Amphibians and Reptiles from the Municipality of Atotonilco el Grande, Hidalgo, México. Herpetologycal Review 47 (1): 91-93

Fernández-Badillo, L., C.R. Olvera-Olvera, S.Y. Valdez-Rentería, F. Torres-Angeles e I. Goyenechea. 2016b. New records of Thamnophis pulchrilatus (Squamata: Natricidae) from the state of Hidalgo, Mexico. Mesoamerican herpetology 3 (2): 519-523

Fernández-Badillo, L., N. Manríquez-Morán, J.M. Castillo-Cerón e I. Goyenechea. 2016c. Análisis herpetofaunístico de la zona árida del estado de Hidalgo. Revista mexicana de Biodiversidad 87 (1): 156-170.

Fernández-Badillo, L., G. Sánchez-Martínez and I. Goyenechea. 2016d. Tropidodipsas sartorii (Cope, 1863). Distribution note. Mesoamerican Herpetology 3 (4): 1061.

Fernández-Badillo, L., S.Y. Valdez-Rentería, C.R. Olvera-Olvera, G. Sánchez-Martínez, N.L. Manríquez-Moran e I. Goyenechea. 2017a. The snake Lampropeltis annulata, Kennicott, 1861, in Hidalgo, Mexico. Mesoamerican Herpetology 4 (2): 502-506.

Fernández-Badillo, L., N. Morales-Capellán, C.R. Olvera-Olvera, G. Montiel-Canales e I. Goyenechea. 2017b. Guía de las serpientes del Estado de Hidalgo. Universidad Autónoma del Estado de Hidalgo. Pachuca, Hidalgo. 272 pp.

Flores-Hernández, M. A., y L. Fernández-Badillo. en preparación. Herpetofauna del Parque Nacional Los Mármoles.

Flores-Villela, O. A., F. Mendoza-Quijano, G. González-Porter. 1995. Recopilación de claves para la determinación de anfibios y reptiles de México. Publicaciones Especiales del Museo de Zoología 10: $1-285$. 
Gelover-Alfaro, A., T. Altamirano-Álvarez y M. Soriano. 2000. Uso de recursos espacio-temporales de la herpetofauna de Metztitlán, Hidalgo. Revista de Zoología 11: 4-11.

Goyenechea, I., V. Vite-Silva y M. E. Mendiola-González. 2010. Boa constrictor. Herpetological Review 41 (4): 515.

Heimes, P. 2016. Snakes of México. Editorial Chimaira. Alemania. 572 pp.

Hernández-Pérez, E. 1997. La herpetofauna de Metztitlán, Hidalgo México: problemática e importancia.

Tesis de Licenciatura. Universidad Nacional Autónoma de México. México. 46 pp.

International Union for Conservation of Nature (IUCN). 2017. The IUCN Red List of Threatened Species. Consultado: 24-11-2016. En http://www.iucnredlist.org

Lemos-Espinal, J.A. y G.R. Smith. 2015. Amphibians and Reptiles of the state of Hidalgo, Mexico. Check List 11 (3): 1-11.

Lemos-Espinal, J. A., y J. R. Dixon. 2016. Anfibios y reptiles de Hidalgo, México. Comisión Nacional para el Conocimiento y Uso de la Biodiversidad (CONABIO). México. 763 pp.

Liner, E.A. y G. Casas-Andreu. 2008. Nombres estándares en español, en inglés y nombres científicos de los anfibios y reptiles de México. Herpetological Circular 38, society for the study of amphibians and reptiles. EUA. 162 pp.

Manzanilla, J. y J. Péfaur. 2000. Consideraciones sobre métodos y técnicas de campo para el estudio de anfibios y reptiles. Revista Ecológica Latino Americana 7 (1-2): 17-30.

Medina-Aguilar, O., J. Alvarado-Díaz y I. Suazo-Ortuño. 2011. Herpetofauna de Tacámbaro, Michoacán, México. Revista Mexicana de Biodiversidad 82 (4):1194-1202.

Mendoza-Quijano, F. 1990. Estudio herpetofaunístico en el transecto Zacualtipán-Zoquizoquipan-San Juan Metztitlán, Hidalgo. Tesis de Licenciatura. Universidad Nacional Autónoma de México. México. 97 pp.

Mendoza-Quijano, F. y Smith H.M. 1993. A New Species of Hooknose Snake, Ficimia (Reptilia, Serpentes). Journal of Herpetology 27 (4): 406-410.

Morales-Capellán, N., L. Fernández-Badillo, A. López-Mejía, G.Sánchez-Martínez e I. Goyenechea. 2016. Confirmation of the nigth snake Hypsiglena tanzeri in Hidalgo, México, and a new record for Reserva de la Biosfera Barranca de Metztitlán. Mesoamerican Herpetology 3 (4): 1097-1100.

Moreno, C. E. 2001. Métodos para medir la biodiversidad. M\&T-Manuales y Tesis SEA, Zaragoza. 84 pp. 
Mulcahy, D. G., and J. R. Mendelson III. 2000. Phylogeography and speciation of the morphologically variable, widespread species Bufo valliceps, base on molecular evidence from mtDNA. Molecular Phylohenetics and Evolution 7 (2):173-189.

Muñoz-Alonso, L. A. 1998. Estudio herpetofaunístico del Parque Estatal Omiltemi, municipio de Chilpancingo, Gro. Tesis de Licenciatura. Universidad Autónoma de México. México. 111 pp. Olson, D. M., E. Dinerstein, E.D. Wikramanayake, N.D. Burges, G.V.N. Powell, E.C. D. Underwood, J.A. Amico, I. Itoua, H.E. Strand, J.C. Morrison, C.J. Loucks, T.F. Allnut, T. H. Ricketts, Y. Kura, J.F. Lamoreux, W.W. Wettengel, P. Hedao, y K.R. Kassem. 2001. Terrestrial Ecoregions of the World: A New Map of Life on Earth. BioScience, 51 (11): 933-938.

Olvera-Olvera, C.R. y J.C. Iturbe-Morgado. 2017. Indotyphlops braminus (Daudin, 1803). Distribution note. Mesoamerican herpetology 4 (1): 201.

Ortiz-Pulido, R., J. Bravo-Cadena, V. Martínez-García, D. Reyes, M.E. Mendiola-González, M. Sánchez y M. Sánchez. 2010. Avifauna de la Reserva de la Biosfera Barranca de Metztitlán, Hidalgo, México. Revista Mexicana de Biodiversidad 81 (2): 373-391.

Pampa-Ramírez, J.T. 2010. Revisión taxonómica de las especies de la familia Leptotyphlopidae en el estado de Hidalgo. Tesis de Licenciatura. Universidad de Guadalajara. México. 49 pp.

Pineda-López, R., y J. R. Verdú-Franco. 2013. Cuaderno de prácticas. Medición de la biodiversidad: diversidad alfa, beta y gama. Universidad Autónoma de Querétaro y Universidad de Alicante. Editorial Universitaria, Colección Académica, Serie Nodos. 114 pp.

Ramírez-Bautista, A., U. Hernández-Salinas, F. Mendoza-Quijano, R. Cruz-Elizalde, B.P. Stephenson, V.D. Vite-Silva y A. Leyte-Manrique. 2010. Lista anotada de los anfibios y reptiles del estado de Hidalgo, México. Universidad Autónoma del Estado de Hidalgo. Pachuca, Hidalgo. 104 pp.

Ramírez-Bautista, A., U. Hernández-Salinas, R. Cruz-Elizalde, C. Berriozabal-Islas, D. LaraTufiño, I. Goyenechea-Mayer y J.M. Castillo-Cerón. 2014. Los anfibios y reptiles de Hidalgo, México: Diversidad, Biogeografía y Conservación. Sociedad Herpetológica Mexicana. Pachuca, Hidalgo. 387 pp.

Rojas-Martínez, A., M. Aguilar-López, C. Cornejo-Latorre, L. G. Juérez-Castillo y S. D. HernándezFlores. 2017. Guía de los mamíferos de la Reserva de la Biosfera Barranca de Metztitlán. Universidad Autónoma del Estado de Hidalgo. Pachuca, Hidalgo. 199 pp. 
Rossman, D. A., N. D. Ford y R. A. Seigel. 1996. The garter snake evolution and ecology. University of Oklahoma Press. EUA. 327 pp.

Rovito, S. M., G. Parra-Olea, E. Recuero y D. B. Wake. 2015. Diversification and biogeographical history of Neotropical plethodontid salamanders. Zoological Journal of the Linnean Society 175 (1): 167-188.

Secretaría de Medio Ambiente y Recursos Naturales (SEMARNAT). 2010. Norma Oficial Mexicana NOM-059-SEMARNAT-2010, Protección ambiental Especies nativas de México de flora y fauna silvestres - Categorías de riesgo y especificaciones para su inclusión, exclusión o cambio -Lista de especies en riesgo. Diario Oficial de la Federación, segunda sección. México. 78 pp.

Smith, H. M. y E.H. Taylor. 1950. An annotated checklist and key to the reptiles of México exclusive of snakes. Bulletin of United States National Museum 199:1-253.

Smith, H.M., y E.H. Taylor. 1966. Herpetology of México. Annotated checklist and keys to amphibians and reptiles. A reprint of Bulletins 187, 194 and 199 of the United States National Museum with a list of subsequent taxonomic innovation. Eric Lundberg Asthon Maryland. EUA. 610 pp.

Uribe-Peña, Z., A. Ramírez-Bautista y G. Casas-Andreu. 1999. Anfibios y reptiles de las serranías del Distrito Federal, México. Universidad Nacional Autónoma de México. México. 111 pp.

Valdez- Rentería. S.Y. 2017. Actualización del conocimiento herpetofaunístico de la Reserva de la Biosfera Barranca de Metztitlán, Hidalgo. Tesis de Licenciatura. Universidad Autónoma del Estado de Hidalgo. México. 98 pp.

Vite-Silva, V. D. 2008. Diversidad de anfibios y reptiles de la Reserva de la Biosfera Barranca de Metztitlán, Hidalgo, México. Tesis de Licenciatura. Universidad Autónoma del Estado de Hidalgo. México. 98 pp.

Vite-Silva, V. D., A. Ramírez-Bautista y U. Hernández-Salinas. 2010. Diversidad de anfibios y reptiles de la Reserva de la Biosfera Barranca de Metztitlán, Hidalgo, México. Revista Mexicana de Biodiversidad 81 (2): 473-485.

Wilson, L.D. y Johnson, J.D. 2010. Distributional patterns of the herpetofauna of Mesoamerica, a biodiversity hotspot. pp. 31-235. En: Wilson, L.D., J.H. Towsend y J.D. Johnson (Eds.). Conservation of the Mesoamerican Amphibians and Reptiles. Eagle Mountain Publisher, L. C. Eagle Mountain, Utah. 


\section{CITA:}

Valdez-Rentería, S.Y., L. Fernández-Badillo, C. R. Olvera-Olvera, G. Sánchez-Martínez, y Goyenechea I. 2018. Listado actualizado de la Herpetofauna de la Reserva de la Biosfera Barranca de Metztitlán, Hidalgo, México. Áreas Naturales Protegidas Scripta, 2018. Vol. 4 (2): 29-48. https://doi.org/10.18242/anpscripta.2018.04.04.02.0002

Sometido: 25 de Mayo de 2018

Revisado: 5 de Junio de 2018

Aceptado: 17 de Septiembre de 2018

Editora asociada: Dra. Cecilia Jiménez Sierra

Idioma Inglés Abstract: Ms.C. Diana Dorantes

Diseño gráfico editorial: Lic. Gerardo Hernández 


\section{Anexo I}

Lista de los anfibios de la RBBM. Los superíndices en las especies corresponden a: $1=$ registrada en campo; $2=$ registrada en la literatura; 3 = registrada en bases de datos; $\bullet=$ nuevo registro para la RBBM. A = Amenazada; $\operatorname{Pr}$ $=$ Bajo protección especial; $\mathrm{Pm}=$ Preocupación menor; $\mathrm{Ca}=$ Casi amenazada; $\mathrm{Vu}=$ Vulnerable; $\mathrm{D}=\mathrm{Distribución}$; $\mathrm{E}=$ Endémico a México.

\begin{tabular}{|c|c|c|c|c|c|}
\hline CLASE Y ORDEN & FAMILIA & ESPECIE & NOM & IUCN & $\mathrm{D}$ \\
\hline Amphibia: Caudata & Plethodontidae & Aquiloeurycea cephalica ${ }^{1} \bullet$ & A & $\mathrm{Ca}$ & $\mathrm{E}$ \\
\hline \multirow[t]{13}{*}{ Amphibia: Anura } & Bufonidae & Incilius occidentalis ${ }^{1,2}$ & & $\mathrm{Pm}$ & $\mathrm{E}$ \\
\hline & & Incilius nebulifer ${ }^{1,2,3}$ & & $\mathrm{Pm}$ & \\
\hline & & Rhinella horribilis ${ }^{1,2,3}$ & & $\mathrm{Pm}$ & \\
\hline & Craugastoridae & Craugastor augusti $i^{1,2}$ & & Pm & \\
\hline & Eleutherodactylidae & Eleutherodactylus verrucipes ${ }^{1} \bullet$ & $\operatorname{Pr}$ & $\mathrm{Vu}$ & $\mathrm{E}$ \\
\hline & Hylidae & Rheohyla miotympanum ${ }^{1,2,3}$ & & $\mathrm{Ca}$ & E \\
\hline & & Dryophytes arenicolor ${ }^{1} \bullet$ & & $\mathrm{Pm}$ & \\
\hline & & Dryophytes eximius ${ }^{1,2}$ & & $\mathrm{Pm}$ & $\mathrm{E}$ \\
\hline & & Dryophytes plicatus ${ }^{1} \bullet$ & A & $\mathrm{Pm}$ & E \\
\hline & Ranidae & Rana berlandieri ${ }^{1,2,3}$ & $\operatorname{Pr}$ & $\mathrm{Pm}$ & \\
\hline & & Rana montezumae, ${ }^{2}$ & $\operatorname{Pr}$ & $\mathrm{Pm}$ & $\mathrm{E}$ \\
\hline & & Rana spectabilis $1,2,3$ & & $\mathrm{Pm}$ & $\mathrm{E}$ \\
\hline & Scaphiopodidae & Spea multiplicata $1,2,3$ & & $\mathrm{Pm}$ & \\
\hline
\end{tabular}


Lista de las tortugas y lagartijas de la RBBM. Los superíndices en las especies corresponden a: $1=$ registrada en campo; $2=$ registrada en la literatura; $3=$ registrada en bases de datos; $\bullet=$ nuevo registro para la RBBM. A= Amenazada; $\mathrm{Pr}=$ Bajo protección especial; $\mathrm{Pm}=$ Preocupación menor; $\mathrm{Vu}=$ Vulnerable; D= Distribución; E= Endémico a México.

\begin{tabular}{|c|c|c|c|c|c|}
\hline CLASE Y ORDEN & FAMILIA & ESPECIE & NOM & IUCN & $\mathbf{D}$ \\
\hline Sauropsida: Testudines & Kinosternidae & Kinosternon hirtipes ${ }^{1} \bullet$ & $\operatorname{Pr}$ & $\mathrm{Pm}$ & \\
\hline \multirow[t]{21}{*}{ Sauropsida: Squamata } & Anguidae & Abronia taeniata $^{1} \bullet$ & $\operatorname{Pr}$ & $\mathrm{Vu}$ & $\mathrm{E}$ \\
\hline & & Barisia imbricata ${ }^{1} \bullet$ & $\operatorname{Pr}$ & $\mathrm{Pm}$ & $\mathrm{E}$ \\
\hline & & Gerrhonotus infernalis ${ }^{2}$ & & $\mathrm{Pm}$ & \\
\hline & & Gerrhonotus liocephalus ${ }^{2}$ & $\operatorname{Pr}$ & $\mathrm{Pm}$ & $\mathrm{E}$ \\
\hline & & Gerrhonotus ophiurus ${ }^{1,2}$ & & $\mathrm{Pm}$ & $\mathrm{E}$ \\
\hline & Gekkonidae & Hemidactylus frenatus ${ }^{1,2}$ & & $\mathrm{Pm}$ & \\
\hline & Phrynosomatidae & Phrynosoma orbiculare ${ }^{1,2}$ & A & $\mathrm{Pm}$ & $\mathrm{E}$ \\
\hline & & Sceloporus grammicus $1,2,3$ & $\operatorname{Pr}$ & $\mathrm{Pm}$ & \\
\hline & & Sceloporus minor ${ }^{1,2}$ & & $\mathrm{Pm}$ & $\mathrm{E}$ \\
\hline & & Sceloporus mucronatus ${ }^{2}$ & & $\mathrm{Pm}$ & $\mathrm{E}$ \\
\hline & & Sceloporus parvus ${ }^{1,2}$ & & $\mathrm{Pm}$ & E \\
\hline & & Sceloporus spinosus $1,2,3$ & & Pm & $\mathrm{E}$ \\
\hline & & Sceloporus torquatus ${ }^{1,2}$ & & $\mathrm{Pm}$ & $\mathrm{E}$ \\
\hline & & Sceloporus variabilis $1,2,3$ & & $\mathrm{Pm}$ & \\
\hline & Scincidae & Plestiodon lynxe $e^{1,2}$ & $\operatorname{Pr}$ & $\mathrm{Pm}$ & $\mathrm{E}$ \\
\hline & & Plestiodon tetragrammus ${ }^{1} \bullet$ & & $\mathrm{Pm}$ & \\
\hline & & Scincella gemmingeri ${ }^{1,2}$ & & $\mathrm{Pm}$ & $\mathrm{E}$ \\
\hline & & Scincella silvícola ${ }^{1,2}$ & $\mathrm{~A}$ & $\mathrm{Pm}$ & $\mathrm{E}$ \\
\hline & Teiidae & Aspidoscelis gularis $1,2,3$ & & $\mathrm{Pm}$ & \\
\hline & Xantusidae & Lepidophyma occulor 1,2 & $\operatorname{Pr}$ & $\mathrm{Pm}$ & E \\
\hline & & Lepidophyma sylvaticum ${ }^{1,2}$ & $\operatorname{Pr}$ & $\mathrm{Pm}$ & E \\
\hline
\end{tabular}




\section{Anexo 2 b}

Lista de las serpientes de la RBBM. Los superíndices en las especies corresponden a: 1=registrada en campo; $2=$ registrada en la literatura; $3=$ registrada en bases de datos; $\bullet=$ nuevo registro para la RBBM. A= Amenazada; $\mathrm{Pr}=$ Bajo protección especial; $\mathrm{Pm}=$ Preocupación menor; $\mathrm{Pe}=$ Peligro de extinción; $\mathrm{Di}=\mathrm{Datos}$ insuficientes; $\mathrm{Vu}=$ Vulnerable; D= Distribución; E= Endémico a México.

\begin{tabular}{|c|c|c|c|c|c|}
\hline CLASE Y ORDEN & FAMILIA & ESPECIE & NOM & IUCN & D. \\
\hline \multirow[t]{33}{*}{ Sauropsida: Squamata } & Boidae & Boa imperator ${ }^{1,2}$ & A & & \\
\hline & Colubridae & Conopsis lineata $^{2}$ & & Pm & E \\
\hline & & Drymarchon melanurus s, $2,3^{2}$ & & Pm & \\
\hline & & Ficimia hardyi ${ }^{1,2}$ & & $\mathrm{Pe}$ & E \\
\hline & & Lampropeltis polyzona $^{2}$ & & & $\mathrm{E}$ \\
\hline & & Lampropeltis annulata ${ }^{1,2}$ & & & $\mathrm{E}$ \\
\hline & & Leptophis mexicanus $^{2}$ & A & Pm & \\
\hline & & Masticophis schotti ${ }^{1,2}$ & & $\mathrm{Pm}$ & \\
\hline & & Oxybelis aeneus ${ }^{1,2}$ & & & \\
\hline & & Pituophis deppei ${ }^{1,2,3}$ & A & Pm & $\mathrm{E}$ \\
\hline & & Salvadora bairdi ${ }^{1} \bullet$ & $\operatorname{Pr}$ & Pm & $\mathrm{E}$ \\
\hline & & Senticolis triaspis $1,2,3$ & & & \\
\hline & & Tantilla bocourti ${ }^{1,2}$ & & Pm & $\mathrm{E}$ \\
\hline & & Tantilla rubra ${ }^{1,2}$ & $\operatorname{Pr}$ & $\mathrm{Pm}$ & \\
\hline & & Trimorphodon tau ${ }^{1,2}$ & & Pm & $\mathrm{E}$ \\
\hline & Dipsadidae & Geophis latifrontalis $^{2}$ & $\operatorname{Pr}$ & $\mathrm{Di}$ & $\mathrm{E}$ \\
\hline & & Hypsiglena tanzeri ${ }^{1,2}$ & & $\mathrm{Di}$ & $\mathrm{E}$ \\
\hline & & Leptodeira maculata ${ }^{2}$ & $\operatorname{Pr}$ & $\mathrm{Pm}$ & $\mathrm{E}$ \\
\hline & & Leptodeira septentrionalis ${ }^{1,2}$ & & & \\
\hline & & Rhadinaea gaigeae ${ }^{1,2}$ & & $\mathrm{Di}$ & $\mathrm{E}$ \\
\hline & & Tropidodipsas sartorii ${ }^{1,2}$ & & & \\
\hline & Elapidae & Micrurus tener ${ }^{1,2,3}$ & $\operatorname{Pr}$ & Pm & \\
\hline & Leptotyphlopidae & Rena dulcis $1,2,3$ & & $\mathrm{Pm}$ & \\
\hline & Natricidae & Nerodia rhombifer ${ }^{1,2}$ & & $\mathrm{Pm}$ & \\
\hline & & Storeria dekayi ${ }^{1,2}$ & & Pm & \\
\hline & & Storeria hidalgoensis ${ }^{1,2}$ & & $\mathrm{Vu}$ & $\mathrm{E}$ \\
\hline & & Thamnophis cyrtopsis ${ }^{1,2}$ & A & Pm & \\
\hline & & Thamnophis proximus ${ }^{1,2,3}$ & A & Pm & \\
\hline & & Thamnophis pulchrilatus ${ }^{1,2}$ & & $\mathrm{Pm}$ & $\mathrm{E}$ \\
\hline & Typhlopidae & Indotyphlops braminus & & & \\
\hline & Viperidae & Crotalus aquilus ${ }^{1,2}$ & $\operatorname{Pr}$ & $\mathrm{Pm}$ & $\mathrm{E}$ \\
\hline & & Crotalus atrox $1,2,3$ & $\operatorname{Pr}$ & $\mathrm{Pm}$ & \\
\hline & & Crotalus molossus $^{1,2}$ & $\operatorname{Pr}$ & Pm & \\
\hline
\end{tabular}

